\title{
Trends in age distribution of participants in a self-covered and a public expense-covered health check-up programs in Japan
}

\author{
Maki Ogawa ${ }^{1}$, Atsushi Imai ${ }^{{ }^{*}}$ \\ ${ }^{1}$ Health Check-Up Registry, Matsunami General Hospital, Kasamatsu, Japan \\ ${ }^{2}$ Institute of Endocrine-Related Cancer, Matsunami General Hospital, Kasamatsu, Japan; \\ *Corresponding Author: aimai@matsunami-hsp.or.jp
}

Received 6 July 2012; revised 4 August 2012; accepted 12 August 2012

\begin{abstract}
Objective: In Japan, there are unique facilities (namely Ningen Dock) of health check-up, where asymptomatic participants undergo a medical examination at their own expense. The earlier occurrence of cervical cancer and the concern on screening prompted us compare to the age distribution in the self-covered system with that of free physical check-up programs at public expense. Methods: We analyzed medical records of Japanese women, who underwent gynecological examinations at self-covered expense and at public expense between for the periods 2002-2011 and 2005-2009, respectively, restricting examinee's age group. Results: For selfcovered system, approximately $80 \%$ of the overall examinee population was occupied with three age groups $30-39,40-49$ and $50-59$. The participants was extremely fewer in the over 60 years age group accounting for $10 \%$, compared to those for the public expense-covered system, the over 60 years age group being $25 \%$. Participant under the age of 30 years seemed to increase in chronological order in both systems. Conclusion: The level of knowledge on sexually transmitted infections may contribute to screening promotion for the younger women, while the elderly over 60 years' attitudes toward screening may be mainly related to social-economic status and/or public expense support.
\end{abstract}

Keywords: Health Check-Up; Cervical Cancer; Sexually Transmitted Disease; Human Papillomavirus; Age Distribution

\section{INTRODUCTION}

Cervical cancer is the second most common malign- nancy among woman worldwide [1,2]. Substantial data point to persistent human papillomavirus (HPV) infection as its cause [3-5]. Organized screening programs for cervical cancer using the cervical smears have been shown to be effective in decreasing mortality and incidence from the disease [6,7]. The cervical screening is a relatively simple, low cost and noninvasive method. Together with transvaginal ultrasonography for detection of ovarian and uterine tumors, a routine cervical screening reduces the probability of developing gynecological malignant diseases.

The most frequently sexually transmitted disease (STD) worldwide is HPV infection. Societies where sexual activity starts at a young age and where multiple partners are common are at a higher risk of exposure to HPV than in conservative societies [8]. In many countries, undergoing cancer screening is not mandatory but voluntary. The level of knowledge and attitude toward screening are related to multiple factors such as ethnicity, place of residence, income, and social-economic status [9-13]. In Japan, there are unique facilities (namely Ningen Dock) of health check-up including gynecological cancer screening activities, where asymptomatic participants undergo a medical examination at their own expense. For low- or mild-income women, local communities support free physical check-up programs, by which asymptomatic women undergo a medical examination at public expense. The aim of this study was to compare the age distribution in the self-covered system with that of public expense-covered physical check-up programs. The gynecological positive findings in these systems have been reported elsewhere [14,15].

\section{METHODS}

We extracted registered cases of cervical cancer screening data from a self-covered health check-up system (Ningen Dock) and a local government expensecovered health check up program databases. The analysis 
was restricted to their age groups.

Between February 2002 and December 2011, 12,783 asymptomatic women, age 18 - 82, visited the Ningen Dock in Matsunami General Hospital for their physical check-up. The women underwent medical evaluations including a medical history, physical examination, blood sampling, urine sampling and radiological imaging as part of a routine health check-up and cancer screening. The cost was not covered by the social insurance. For free physical check-up systems, between January 2005 and December 2009, 2850 asymptomatic women, age 21 82, visited one of 4 Kasamatsu Community-agreed gynecologic physician for their physical check-up. The cost was fully covered by the municipal corporation.

\section{RESULTS}

Figures 1 and $\mathbf{2}$ demonstrate the age distribution of the participants of self-covered check-up system (Ningen Dock) and public expense-covered check-up program, respectively. For self-covered check-up system, all five chronological groups of examinees had a peak at the ages of 40 - 49 (Figure 1). Approximately $80 \%$ of the overall examinee population was occupied with three age groups 30 - 39, 40 - 49 and 50 - 59. This accounted for 5 to $10 \%$ in over 60 years age group. For public expensecovered system (Figure 2), the participants were distributed almost equally in age groups 30 - 39 through 60 - 69 . Over 25\% was in over 60 years age group.

In the younger group ( $<29$ years), the participants increase in chronological order in both systems (Figures 1 and 2).

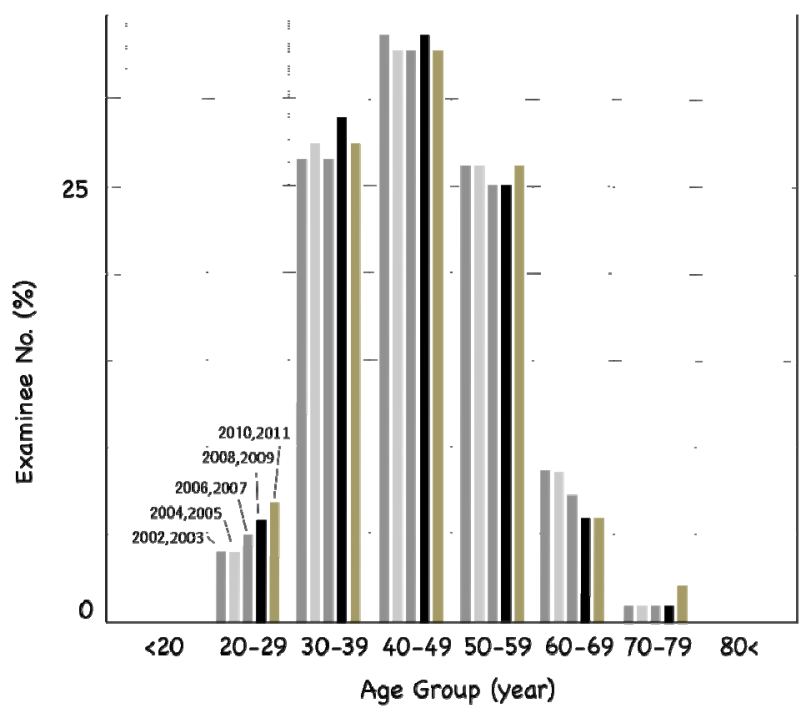

Figure 1. Examinee number distributed by age class of a self-covered health check-up system (Ningen Dock) for the years 2002 through 2010. The values are expressed as percentage of the overall examinee population (2354 for the years 2002 and 2003, 2246 for 2004 and 2005, 2986 for 2006 and 2007, 2323 for 2008 and 2009, 2874 for 2010 and 2011).

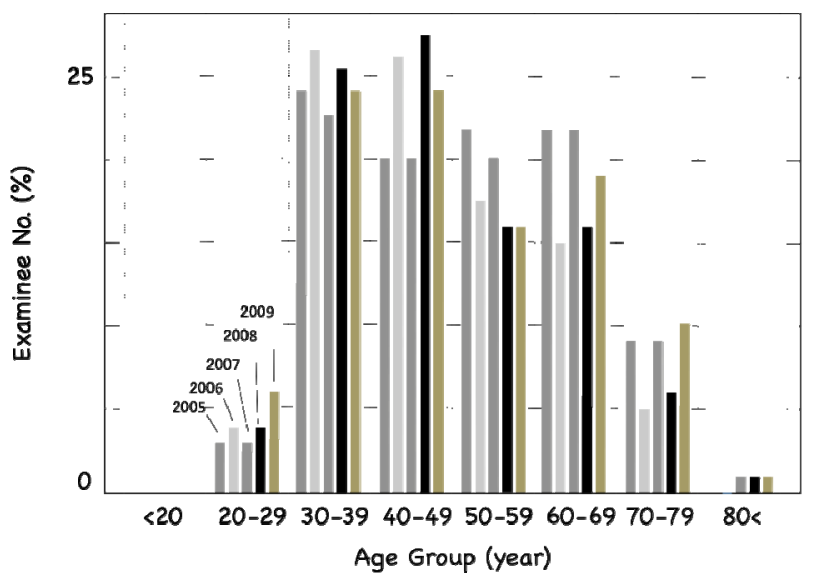

Figure 2. Examinee number distributed by age class of a public expense-covered health check-up system for the years 2005 through 2009. The values are expressed as percentage of the overall examinee population (922 for the year 2005, 207 for 2006, 714 for 2007, 224 for 2008, and 776 for 2009).

\section{DISCUSSION}

Substantial data point to persistent human papillomavirus (HPV) infection, most frequently STD, as cervical cancer cause. The mean time between HPV infection and invasive cancer is about 15 years, and within 2 to 4 years of detection $15.5 \%$ to $25.5 \%$ of low-grade epithelial lesions become high-grade lesions [3-5]. Societies where sexual activity starts at a young age and where multiple partners are common are at high risk of exposure to HPV than in conservative societies. For example, a study in Jordan, one of the most conservative and religious countries, found the very low incidences of dysplastic changes and cervical cancer [8]. In Ningen Dock which medical records we have analyzed, asymptomatic participants undergo a medical examination at their own expense [14]. The cultural tradition and high concern on check-up of our subjects restrict the likelyhood of multiple sexual partners. This may explain why very low incidence of dysplastic changes and cervical cancer were found in our study group of women. Moreover, our previous study on community-agreed check-up, where asymptomatic women visited community and migrant health centers at public expense [15], also has shown lower incidence of malignancy diseases. Public support can improve cancer screening rates among women with high-attitude toward screening but non-high income.

In the present study, which was based on the records from physical check-up system where asymptomatic persons undergo a medical examination at their own expense or at public expense, particular attention such as public expense may provide the elderly above 60 years a better attitude towards cancer screening. The level of knowledge on the relationship between sexually transmitted infections and earlier occurrence of cervical can- 
cer may contribute to screening promotion for the younger women.

\section{REFERENCES}

[1] Grangé, G., Malvy, D., Lançon, F., Gaudin, A., El Hasnaoui, A. (2008) Factors associated with regular cervical cancer screening. International Journal of Gynaecology and Obstetrics, 102, 28-33.

doi:10.1016/j.ijgo.2008.01.027

[2] Duarte-Franco, E. and Franco, E. (2004) Cancer of the Uterine Cervix. BMC Womens Health, 4, S13. doi:10.1186/1472-6874-4-S1-S13

[3] Rocha-Zavaleta, L., Yescas, G., Cruz, R.M. and CruzTalonia, F. (2004) Human papillomavirus infection and cervical ectopy. International Journal of Gynaecology and Obstetrics, 85, 259-266. doi:10.1016/j.ijgo.2003.10.002

[4] Tachezy, R., Saláková, M., Hamsíková, E., Kanka, J., Havránková, A. and Vonka, V. (2003) Prospective study on cervical neoplasia: Presence of HPV DNA in cytological smears precedes the development of cervical neoplastic lesions. Sexually Transmitted Infections, 79, 191196. doi:10.1136/sti.79.3.191

[5] Muñoz, N., Bosch, F., de Sanjosé, S., Herrero, R., Castellsagué, X., Shah, K., Snijders, P. and Meijer, C. (2003) Epidemiologic classification of human papillomavirus types associated with cervical cancer. New England Journal of Medicine, 348, 518-527. doi:10.1056/NEJMoa021641

[6] Anttila, A., Ronco, G., Clifford, G., Bray, F., Hakama, M., Arbyn, M. and Weiderpass, E. (2004) Cervical cancer screening programmes and policies in 18 European countries. British Journal of Cancer, 91, 935-941.

[7] Nieminen, P., Kallio, M., Anttila, A. and Hakama, M. (1999) Organised vs spontaneous pap-smear screening for cervical cancer: A case-control study. International Journal of Cancer, 83, 55-58.

doi:10.1002/(SICI)1097-0215(19990924)83:1<55::AID-I JC11>3.0.CO;2-U
[8] Malkawi, S., Abu Hazeem, R., Hajjat, B. and Hajjiri, F. (2004) Evaluation of cervical smears at King Hussein Medical Centre, Jordan, over three and a half years. Eastern Mediterranean Health Journal, 10, 676-679.

[9] Dietrich, A., Tobin, J., Cassells, A., Robinson, C., Greene, M., Sox, C., Beach, M., DuHamel, K. and Younge, R. (2006) Telephone care management to improve cancer screening among low-income women: A randomized, controlled trial. Annals of Internal Medicine, 144, 563571.

[10] Lawson, H., Henson, R., Bobo, J. and Kaeser, M. (2000) Implementing recommendations for the early detection of breast and cervical cancer among low-income women. MMWR Recommendations and Reports, 49, 37-55.

[11] Ng, E., Wilkins, R., Fung, M. and Berthelot, J. (2004) Cervical cancer mortality by neighbourhood income in urban Canada from 1971 to 1996. Canadian Medical Association Journal, 170, 1545-1549. doi:10.1503/cmaj.1031528

[12] Schoenberg, N., Hopenhayn, C., Christian, A., Knight, E. and Rubio, A. (2005) An in-depth and updated perspective on determinants of cervical cancer screening among central Appalachian women. Women Health, 42, 89-105. doi:10.1300/J013v42n02_06

[13] Yabroff, K., Lawrence, W., King, J., Mangan, P., Washington, K., Yi, B., Kerner, J. and Mandelblatt, J. (2005) Geographic disparities in cervical cancer mortality: What are the roles of risk factor prevalence, screening, and use of recommended treatment? Journal of Rural Health, 21, 149-157. doi:10.1111/j.1748-0361.2005.tb00075.x

[14] Imai, A., Matsunami, K., Takagi, H. and Ichigo, S. (2012) Trend of incidence in positive cervical smears from 20022010 in Ningen Dick, a special Japanese health check-up system. Ningen Dock, 26, 923-926.

[15] Takagi, H., Ichigo, S., Matsunami, K. and Imai, A. (2011) Evaluation of a public expense-covered gynecologic screening program in Japan 2005-2009. Open Journal of Obstetrics and Gynecology, 1, 21-24. doi:10.4236/ojog.2011.12005 